heparin prophylaxis. On the postoperative 6th day, neck and shoulder pain were worsened unless she took any anti-inflammatory drug. In the cervical and thoracic MRI, there was a hematoma was detected. Intravenous hydration and antiinflammatory therapy were organized. The neck and shoulder pain on the second day of his second admission decreased dramatically.

Conclusions The most important thing to mention is that PDDH can mask the hematoma-induced headache. It should be kept in mind that a spinal hematoma can be observed even VTE prophylaxis was started according to guidelines.

\section{CONTINUOUS SPINAL ANESTHESIA AS A SAFE ALTERNATIVE FOR RETROPUBIC PROSTATECTOMY IN A HIGH-RISK PATIENT}

P Rebelo, M Cunha, C Pereira*. Hospital Pedro Hispano, Matosinhos, Portugal

\subsection{6/rapm-2021-ESRA.25}

Background and Aims Anesthetic management of patients with pulmonary hypertension $(\mathrm{PH})$ is complex and carries a high potential of perioperative morbidity and mortality.

Methods We report a continuous spinal anesthesia (CSA) for retropubic prostatectomy.

Results A 71-year-old man, ASA IV with a very severe COPD conditioning severe $\mathrm{PH}$ was proposed for retropubic prostatectomy. Because of the risks of general anesthesia (GA) in this patient, we decided to perform a CSA with the IntraLong kit from PAJUNK. In lateral position under full aseptic precaution, a $21 \mathrm{G}$ pencil point needle was inserted into the L3-L4 space. After obtaining free flow of cerebrospinal fluid, a $25 \mathrm{G}$ catheter was introduced. We started with $5 \mathrm{mg}$ of levobupivacaine. With a light slope the dose was titrated $(2,5 \mathrm{mg}$ at the time) until adequate block was achieved. During surgery, the patient was always hemodynamically stable and we used a total of $15 \mathrm{mg}$ of levobupivacaine.

At the end of the surgery the catheter was withdrawn, and postoperative recovery was uneventful.

Conclusions The anesthetic management of a patient with $\mathrm{PH}$ is a challenge. GA with positive pressure ventilation in associated with a potential risk of cardiorespiratory decompensation. However, spinal anesthesia is associated with a risk of severe hypotension which can be catastrophic in a patient with low functional capacity. With the use of CSA we can titrate the local anesthetic to maintain hemodynamic stability in the perioperative period with no vasopressors needed and with spontaneous ventilation with a peripheral saturation $>92 \%$.

\section{ANAESTHETIC CONSIDERATIONS: A CASE OF MCCUNE- ALBRIGHT SYNDROME POSTED FOR OPTIC NERVE DECOMPRESSION - A CASE REPORT}

${ }^{1} \mathrm{R}$ Kashyap, ${ }^{2} \mathrm{~K}$ R*, ${ }^{2} \mathrm{D}$ Goswami. ${ }^{1}$ Chandra Laxmi Hospital, Vaishali, India; ${ }^{2}$ All India Institute of Medical Sciences, New Delhi, India

\subsection{6/rapm-2021-ESRA.26}

Background and Aims Mccune Albright syndrome (MAS) consists of a triad of Fibrous dysplasia, café-au-lait macules and autonomous hyperfunctoning endocrinopathies, caused by mutation in $G_{s} \alpha$ subunit of G-protein coupled receptors (GPCRs). Anaesthetic management of these patients could be complicated by difficult airway, increased risk of perioperative arrhythmias, other manifestations of associated endocrinopathies and pathological fractures.

Methods We present the anaesthetic management of a 13 years old boy, case of MAS posted for optic nerve decompression. He had history of multiple fractures involving upper and lower limbs. Drug history included tab carbimazole for hyperthyroidism, calcitriol, calcium and sodium acid phosphate for dysplastic bone. Airway examination revealed coarse facial features with large head and tongue with restricted neck movement towards the right side. NCCT orbit revealed narrowed optic canal secondary to expanded dysplastic bone, causing vision loss in right eye. On the day of surgery, radial artery cannulation was done under local anaesthesia. Intravenous induction was done with fentanyl, propofol and atracurium along with dexmedetomidine infusion. C-Mac video laryngoscope and bougie guided intubation done.

Results Anaesthesia was maintained with oxygen, nitrous oxide, isoflurane, atracurium boluses and dexmedetomidine infusion. Patient positioning was done with extreme care. Paracetamol, ketorolac and morphine were given for pain control. Surgery was uneventful. The patient was shifted to ICU for postoperative monitoring and extubated the next day.

Conclusions Throughout surgery, the patient had increased requirement of anaesthetic agents. GPCRs have been considered as potential targets of inhalational anaesthetics, whether this could have an implication on patients with MAS needs further research.

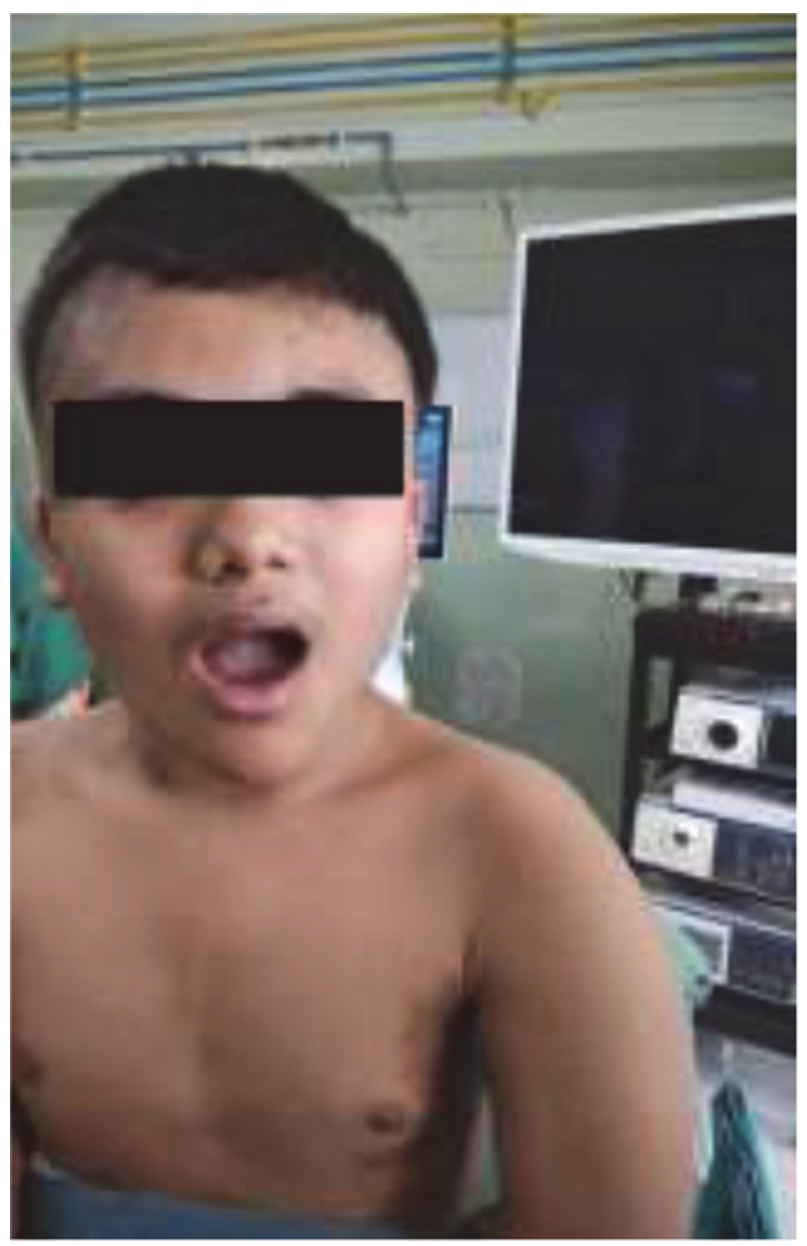

Abstract 26 Figure 1 


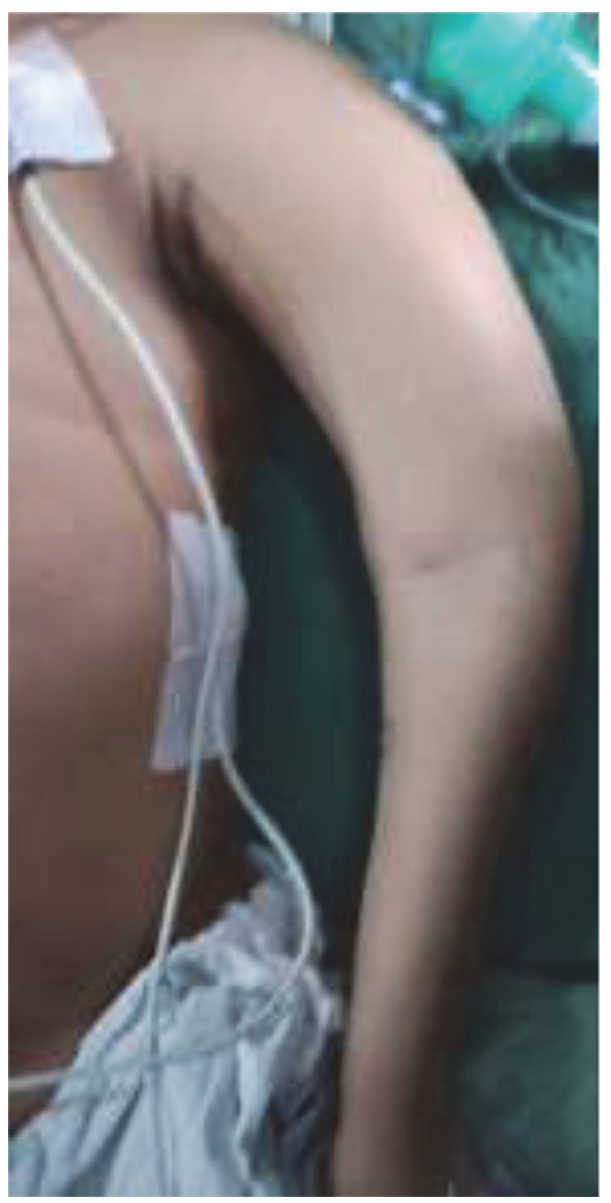

Abstract 26 Figure 2

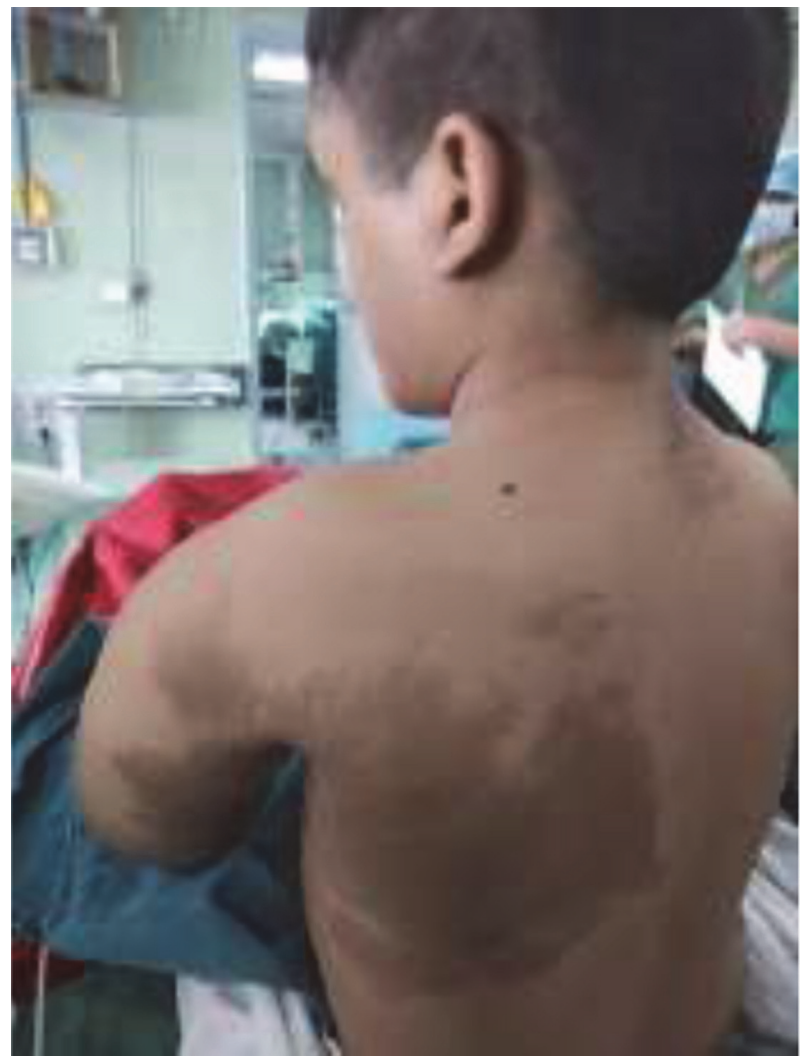

Abstract 26 Figure 3

\section{ANAESTHETIC MANAGEMENT IN PARTURIENT WITH COARCTATION OF AORTA, PATENT DUCTUS ARTERIOSUS AND ARNOLD CHIARI MALFORMATION FOR ELECTIVE CAESAREAN SECTION}

L Aishwarya*, RS Mane, MC Patil. Jawaharlal Nehru Medical College, KLE University, Belagavi, India

\subsection{6/rapm-2021-ESRA.27}

Background and Aims Coarctation of aorta represents 6\%-8\% of CHD with associated Patent ductus arteriosus. Arnold chiari malformation is characterized by prolapse of cerebellar tonsils below the foramen magnum causing compressive symptoms. The primary goal is to minimize the incidence of haemodynamic stressor response and brainstem herniation which is a possible risk with endotracheal intubation.

Methods A case of 24year G2P1L0 with 34weeks POG, a known case of ACM was diagnosed with COA and PDA. She was planned for elective caesarean due to uncontrolled upper limb hypertension. Examination revealed pansystolic murmur and Loud P2 with suzzman's sign positive. She had feeble femoral pulse with radiofemoral delay. Uppler limb BP : 190/ 100 mmhg and lowerlimb BP: 130/80 mmhg. 2DEcho revealed Large PDA with left to right shunt, dilated RA, RV. Severe COA with PPG 76 mmh. Trivial TR with PPG 40 mmhg. No sensory and motor deficits noted. Graded epidural anaesthesia was administered.

Results Parturient with congenital anomalies has been successfully managed perioperatively with graded epidural doses and by providing adequate post-operative analgesia.

Conclusions Parturient with Coarctation of the aorta and Arnold chiari malformation presents with unique challenges to the anaesthetist and management must be tailored to avoid hemodynamic instability and associated risk of tonsillar herniation. The use of epidural anaesthesia in graded dose was successful in achieving this goal.

\section{LOCAL ANAESTHETIC SYSTEMIC TOXICITY AFTER AXILLARY BRACHIAL PLEXUS BLOCK IN AMBULATORY SURGERY - A CASE REPORT}

${ }^{1} \mathrm{GS}$ Sousa*, ${ }^{2} \mathrm{M}$ Oliveira, ${ }^{2} \mathrm{P}$ Esperança, ${ }^{3} \mathrm{E}$ Segura Grau. ${ }^{1} \mathrm{C}$ entro Hospitalar Tondela-Viseu, Viseu, Portugal; ${ }^{2}$ Centro Hospitalar Universitário São João, Porto, Portugal; ${ }^{3}$ Anesthesiology, Centro Hospitalar Tondela-Viseu, Viseu, Portugal

\subsection{6/rapm-2021-ESRA.28}

Background and Aims Local anaesthetic systemic toxicity (LAST) is a rare but potentially fatal complication of regional anaesthesia. LAST affects two major systems, namely neurologic and cardiovascular ${ }^{1}$. The number of regional anaesthesia has been increasing and the risk of LAST, despite all the good practices, increases proportionally. Axillary brachial plexus block is reported as the most associated block to LAST events. $^{2}$

We pretend to increase awareness to this rare but lifethreatening entity.

Methods Case report and literature review.

Results A 55-years-old female patient, ASA II, was admitted for left hand ambulatory surgery. It was performed an ultrasound-guided axillary brachial plexus block. The left axillary artery and the branches of median, radial and cubital nerves were identified. Then $200 \mathrm{mg}$ of mepivacaine and $37.5 \mathrm{mg}$ of levobupivacaine were administered through an in-plane 